\title{
Synchronous Mucinous Tubular and Spindle Cell Carcinoma of the Kidney and Papillary Carcinoma of the Thyroid
}

\author{
Gwendolyn Fernandes ${ }^{1 *}$, Varsha Dipak Deshpande², Sujata Choudhary ${ }^{1}$, Swati Shinde ${ }^{1}$ \\ ${ }^{1}$ Dept of pathology, G.S. Medical College and K.E.M. Hospital, Parel, Mumbai, India \\ ${ }^{2}$ Dept of Uropathology, G.S. Medical College and K.E.M. Hospital, Parel, Mumbai, India
}

\begin{abstract}
A 55 year old female, presented with flank pain and fullness since 2 years. On examination, a 10x10cm, ballotable lump was present in the right flank. CT Abdomen showed a $9.5 \times 8.8 \mathrm{~cm}$, exophytic mass lesion in the right kidney with patchy arterial enhancement and heterogenous enhancement in the venous phase. Whole body PET CT showed well defined, metabolically active masses in the right kidney, thyroid and cervical lymph nodes. Nephrectomy specimen received, showed a tumor measuring $12 \times 7 \times 6.5 \mathrm{~cm}$ creamy-white, fleshy, replacing the kidney. Histopathology revealed an unusual renal neoplasm with tubule formation merging with bland spindle cells and a focal myxoid stroma. Tumor cells were immunoreactive for CK7, EMA, vimentin and were negative for PAX8, CD10 \& AMACR. The diagnosis of Mucinous Tubular and Spindle cell carcinoma (MTSCC) was established. FNAC of the thyroid nodules showed a classic papillary thyroid carcinoma.
\end{abstract}

Keywords: Mucinous Tubular Spindle Cell Carcinoma, Kidney Tumors, Classic Papillary Thyroid Carcinoma, Nephrectomy

\section{Introduction}

MTSCC are rare renal epithelial neoplasms with low malignant potential and with characteristic histomorphological features. MTSCC was first recognized as a specific entity in the WHO classification of $2004^{[1]}$ and also included in the recent version of $2016^{[2]}$. It is seldom reported to be associated with other malignancies. We report a case report of synchronous MTSCC of the kidney with classic papillary carcinoma of the thyroid gland, in a middle-age female patient.

\section{Case Report}

A-55-year old woman presented with flank pain and fullness since 2 years. On examination, a 10x10 cm ballotable lump was present in the right flank. CT abdomen showed a $9.5 \times 8.8 \mathrm{~cm}$, exophytic mass in the right kidney with patchy arterial enhancement and heterogenous enhancement in the venous phase. Whole body PET CT scan revealed well defined, metabolically active masses in the right kidney, thyroid gland and supraclavicular lymph nodes. USG of the neck showed multiple (5-6) subcm to $\mathrm{cm}$, iso to hypoechoic nodules in the right lobe and isthmus of the thyroid gland. Few of these nodules contained microcalcifications within. Right lower lobe nodule also contained curvilinear macrocalcifications with central and peripheral vascularity (TI-RADS 4 score). Mildly enlarged pretracheal, paratracheal and hilar lymph nodes were seen. MGIT and GENE XPERT MTB RIF of mediastinal lymph node aspirate were negative. A right radical nephrectomy was done and her post-operative period was uneventful.
We received a radical nephrectomy specimen with a tumor measuring $12 \times 7 \times 6.5 \mathrm{~cm}$, creamy-white, fleshy, replacing the kidney and involving the pelvicalyceal system. The renal capsule was invaded by tumor at the hilum and tumor was seen to extend into the renal sinus. Gerotas fascia as well as the renal vein, renal artery and ureter were not involved by the tumor. No hilar lymph nodes were seen. On microscopy, the tumor showed characteristic biphasic architecture and was composed of bland tubular structures with abrupt transition to benign spindle cells. Individual tumor cells forming the tubules were cuboidal or oval shaped and showed round to oval nuclei with vacuolated cytoplasm. Mitotic activity was inconspicuous and no abnormal mitoses were noted. The tumor also showed focal intracellular mucin and a myxoid stroma. Few clusters of foamy macrophages were also seen. Alcian blue and PAS stains highlighted the extracellular mucin. Immunohistochemistry was done. The tubular component of the tumor was positive for CK-cocktail while the spindle cell component showed immunoreactivity to vimentin. EMA was focally positive. PAX-8, AMACR, synaptophysin, chromogranin and CD10 were negative.

FNAC from the thyroid isthumus showed classic papillary carcinoma of thyroid (Bethesda category VI). The cytology smears were cellular and composed of papillaroid configurations of thyroid follicular cells. These configurations were monolayered with polygonal cells with anisonucleosis, pale nuclei and prominent intranuclear grooves as well as inclusions. Cervical lymph node status was not known. 
A diagnosis of synchronous mucinous tubular spindle cell carcinoma of the kidney with papillary carcinoma of the thyroid was established.

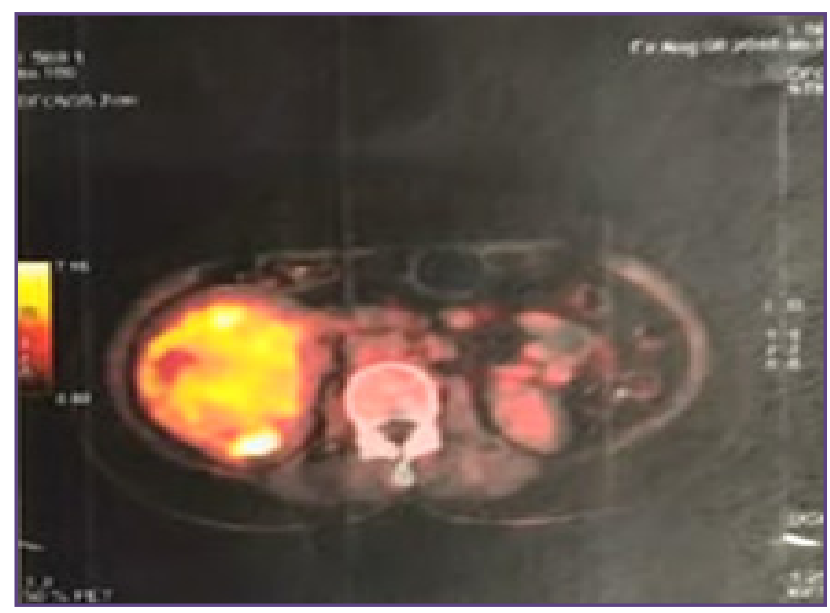

Fig. 1: Whole body PET - well defined, metabolically active masses in the right kidney (Figure 1), thyroid and cervical lymph nodes.

\section{Discussion}

MTSCC is an unusual renal neoplasm characterized by tubule formation showing abrupt transition into bland

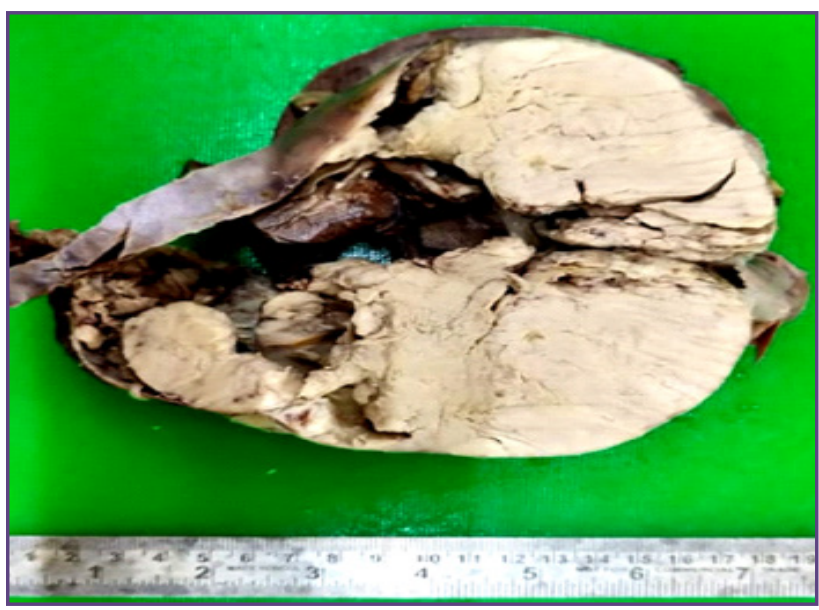

Fig. 2: Tumor measured $12 \times 7.5 \times 6 \mathrm{~cm}$ with creamy-white, fleshy appearance.

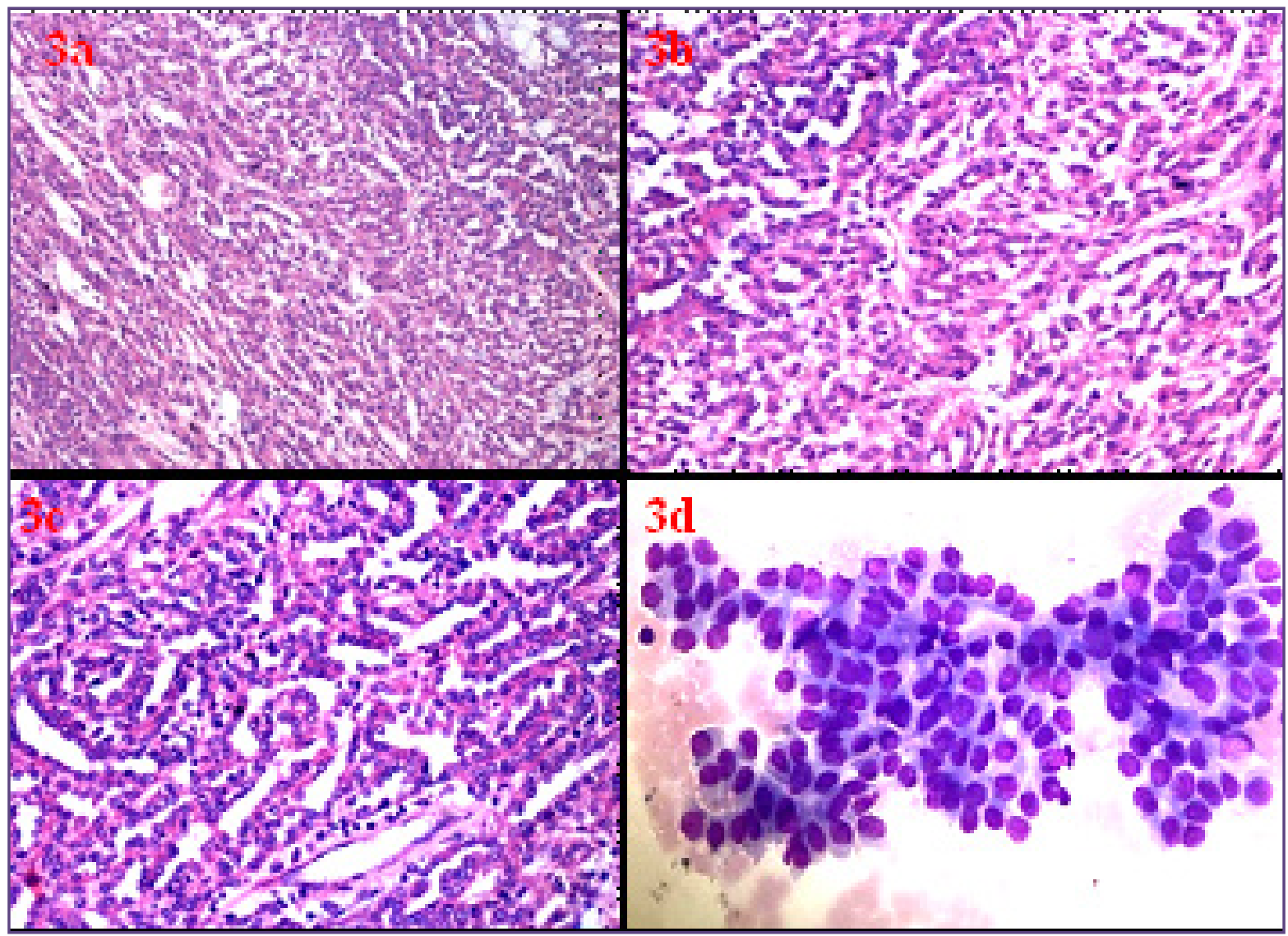

Fig. 3a: Biphasic tumor with tubular and spindle cell components (H\&E X100); 3b: Biphasic tumor with tubular and spindle cell components (H\&E X400); 3c: Tubules composed of cuboidal cells with bland nuclear features (H\&E X 400); 3d: classic papillary carcinoma of the thyroid gland, arrow showing intranuclear inclusion. 


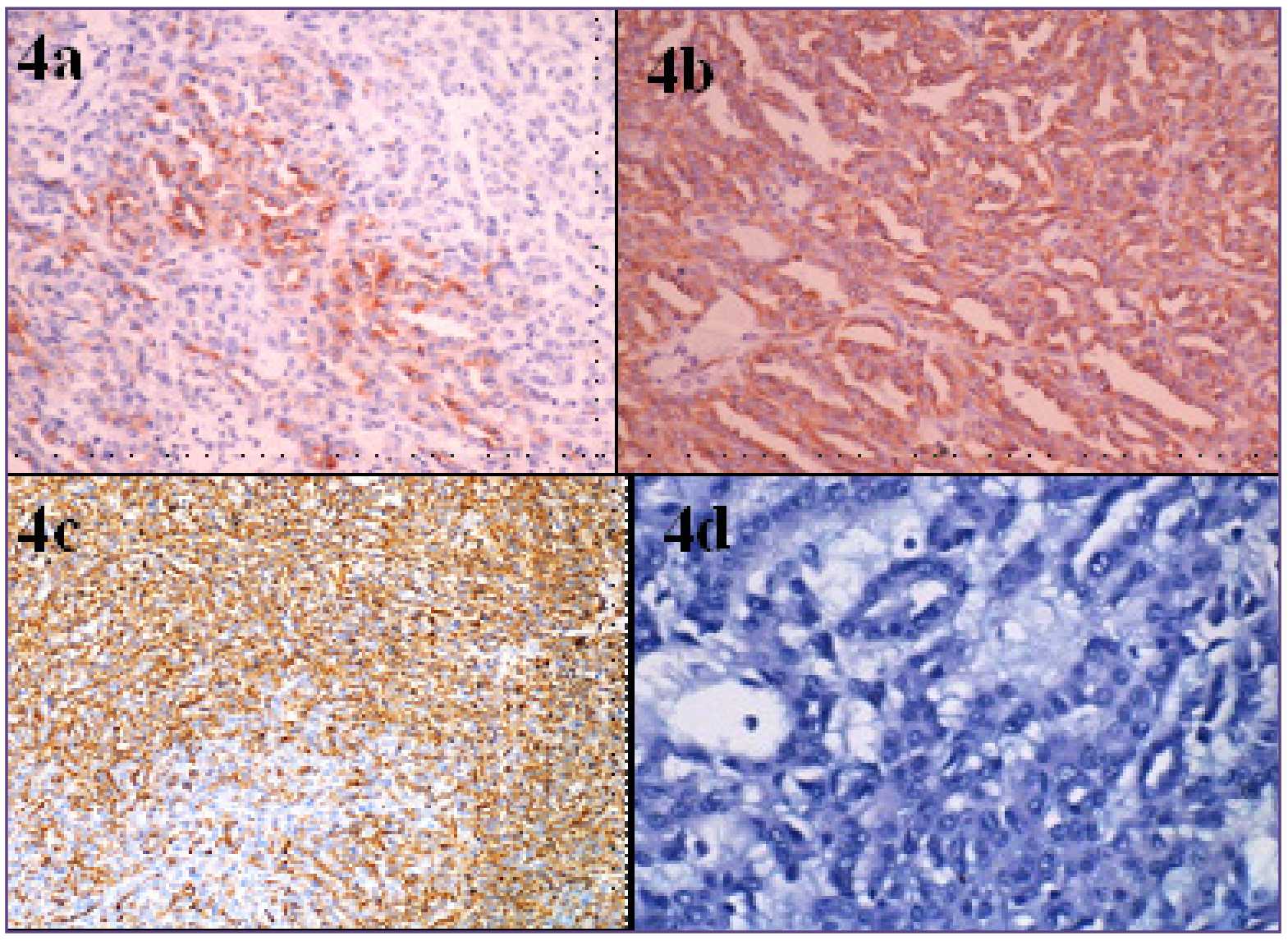

spindle cells and a myxoid stroma. It accounts for $<1 \%$ of all renal tumors and has a relatively good prognosis. It was first recognized and described by Mac in1997 and then by Sringley et al in 1999. ${ }^{[1]}$ It has been recognized as a distinct renal cell tumor in the 2004 WHO classification of renal tumors, and the name MTSCC is self explanatory, based on histomorphological features. It has a wide age range of occurrence from 13 to 81 years, with an average age of 58 years and a female to male ratio of $3: 1$.

The origin of MTSCC remains uncertain and controversy exists about its origin either from proximal convoluted tubules or distal convoluted tubules. Epithelial markers like AMACR, CK7, EMA and vimentin show positivity in $80-100 \%$ cases and favor a distal tubular origin. However, AMACR is also seen in proximal convoluted tubules. ${ }^{[3]}$ Some tumors are found to occur in association with nephrolithiasis.

Grossly, these are well circumscribed, yellow to tan-brown tumors. The histomorphological features are characteristic and may not need IHC work up. These tumors have bland nuclear features but occasionally high grade tumors and tumors with sarcomatoid change have been described.
These tumors usually show positivity for CK7, PAX2 and AMACR. Our case showed PAX2 and AMACR negativity while $\mathrm{CK}$ cocktail and vimentin showed strong positivity. EMA was focally positive.

The commonest differential diagnosis for MTSCC is papillary renal cell carcinoma type 1 with sarcomatoid differentiation and metanephric adenoma. Papillary renal cell carcinoma shows solid or papillary growth pattern with elongated tubules, lacks mucinous stroma and generally shows CD10 positivity. Metanephric adenomas, on the other hand, are smaller in size; do not show myxoid stroma or spindle cells and usually show positivity for WT1 and CD57.

Metastasis to lymph nodes by the high grade tumors have been described ${ }^{[4]}$ and few cases with typical bland morphology with metastasis have also been described. ${ }^{[5]}$

MTSCC is said to show relationship to papillary carcinoma of the kidney on histomorphology as well as immunohistochemistry profile. On genetic profiling, MTSCC lacks the consistent gains of chromosome 7 $\& 17$ and losses of chromosome $\mathrm{Y}^{\left[{ }^{[6]}\right.}$ which are typical of papillary carcinoma of the kidney. MTSCC also 
shows multiple chromosomal losses, including loss of chromosomes $1,4,6,8,9,13,14,15 \& 22$ but none of these are consistent and diagnostic. ${ }^{[6,7]}$

Prognoses of these cases are relatively good, and one year after follow- up, our patient too has no recurrence or metastasis of MTSCC. Surgery for the papillary carcinoma of the thyroid was contemplated a number of times, but has not yet been done due to personal reasons.

MTSCCs do not generally show association with other tumors. Occasional case report of an association with lung adenocarcinoma ${ }^{[8]}$ and ganglioneuroma ${ }^{[9]}$ has been reported. No case reports of synchronous MTSCC with classic papillary carcinoma of the thyroid have been found in English literature, and to the best of our knowledge, this is the first reported case.

\section{Conclusion}

This is an unusual case with two synchronous malignanciesMTSCC of the kidney and classic papillary carcinoma of the thyroid gland. Genetic studies are required to see if there is a co-relation between these two malignancies.

\section{Abbrevations and symbols:}

MTSCC: Mucinous Tubular and Spindle Cell Carcinoma

TI-RADS: Thyroid imaging reporting and data system

PAS: Periodic acid-Schiff

AMACR: Alpha-methylacyl-CoA racemase

MGIT: Mycobacteria Growth Indicator Tube

\section{References}

1. Sringley R. Mucinous tubular and spindle cell carcinoma. In: Eble JN, Sauter G, Epstein JI, Sesterhenn IA, editors. World health organization classification of tumours: pathology and genetics. Tumours of urinary system and male genital organs. 4th ed. IARC Press, Lyon; 2004.

2. Kuroda N, Paner G, Srigley JR. Mucinous tubular spindle cell carcinoma. In: Holger Moch, Humphrey PA, Ulbright TM, editors. Pathology and genetics of tumours of the urinary system and male genital organs. 4th ed. IARC Press, Lyon, France; 2016.

3. Ferlicot S, Allory Y, Comperat F, et al. Mucinous tubular spindle cell carcinoma: a report of 15 cases and a review of literature. Virchows Arch. 2005; 447(6):978-983.

4. Thway K, du Parcq J, Larkin JM, et al. Metastatic renal mucinous tubular and spindle cell carcinoma. Atypical behavior of a rare, morphologically bland tumor. Ann Diagn Pathol. 2012; 16:407-410. PMID:21684183

5. Simon RA, di Sant'agnese PA, Palapattu GS, et al. Mucinous tubular and spindle cell carcinoma of the kidney with sarcomatoid differentiation. Int J Clin Exp Pathol. 2008; 1:180-184. PMID:18784804

6. Cossu-Rocca P, Eble JN, Delahunt B, et al. Mucinous tubular spindle cell carcinoma lacks the gains of chromosomes 7 and 17 and losses of chromosome $\mathrm{Y}$ that are prevalent in papillary renal cell carcinoma. Mod Pathol. 2006; 19: 488-493.

7. Rakozy C, Schmahl GE, Bogner S, Storkel S. Lowgrade tubular-mucinous renal neoplsms: morphologic, immunohistochemical and genetic features. Mod Pathol. 2002; 15:1162-1171. PMID:12429795

8. Park SY, Kang GH, Black J, et al. Mucinous tubular spindle cell carcinoma of the kidney occurring in a patient with pulmonary adenocarcinoma. The Korean Journal of Pathology. 2008; 42:54-59.

9. Chien-Chang Li, Chen-Hui Lee, Yee-Jee Jan, et al. Renal mucinous tubular and spindle cell carcinoma with an aortocaval mass mimicking metastatic lymphadenopathy. Journal of the Chinese Medical Association. 2012;75:240-242.

*Corresponding author:

Dr. Gwendolyn Fernandes, C-802, Swayam, Poonam Gardens, Mira Road, Thane, Maharashtra. INDIA

Phone: +91 9819218405

Email: drgwenfern@yahoo.co.in

Financial or other Competing Interests: None. 